\title{
Knowledge about vaccines and vaccination in older people: Results of a national survey by the Italian Society for Gerontology \& Geriatrics.
}

Fiona Ecarnot ${ }^{1}$, Claudio Pedone ${ }^{2}$, Matteo Cesari ${ }^{3}$, Stefania Maggi ${ }^{4}$, Raffaele Antonelli Incalzi ${ }^{5}$.

${ }^{1}$ Department of Cardiology, University Hospital Besancon, and EA3920, University of Franche-Comté, Besancon, France

${ }^{2}$ Unit of Geriatrics, Università Campus Bio-Medico di Roma, Rome, Italy.

c.pedone@unicampus.it

${ }^{3}$ Fondazione IRCCS Ca' Granda Ospedale Maggiore Policlinico, Milano, Italy; Geriatric Unit, Department of Clinical Sciences and Community Health, University of Milan, Milan, Italy.

${ }^{4} \mathrm{CNR}$, Institute of Neuroscience - Aging Branch, Padua, Italy

${ }^{5}$ Unit of Geriatrics, Università Campus Bio-Medico di Roma, Rome, Italy. r.antonelli@unicampus.it

\section{Address for correspondence:}

Fiona Ecarnot (ORCID ID 0000-0002-4224-9731)

Department of Cardiology

University Hospital Jean Minjoz

3 Boulevard Fleming

25000 Besancon

France

Tel: +33.381.668.539; Fax: +33.381.668.582; Email: Fiona.ecarnot@univ-fcomte.fr 


\section{Introduction}

3 Vaccine preventable diseases (VPDs) represent a major public health issue and a significant burden

4 on healthcare systems, particularly among older persons. Diseases such as seasonal influenza,

5 pneumococcal pneumonia and herpes zoster (HZ) account for considerable morbidity and mortality,

6 and their incidence and mortality increase significantly with age [1-4]. Effective vaccines exist

7 against all these diseases, but uptake of vaccines fails to reach target levels in many regions [1, 2, 5-

$8 \quad 8]$.

9 Vaccine uptake is a complex and multidimensional phenomenon that is the combination of the

10 physician's propensity to offer the vaccine, and the individual's propensity to accept it. Reported

11 physician barriers to offering vaccination include competing demands on the physician's time [9],

12 forgetting to do so during the consultation [10], the cost to the patient [11], as well as low awareness

13 of vaccines and of the recommended target groups [12]. Healthcare providers have been shown to

14 be more aware of disease burden for VPDs, and have more confidence in the efficacy of

15 vaccination, whereas patients often do not feel a need for the vaccine if they don't perceive the

16 disease as a threat to themselves [13]. The perceived severity and incidence of herpes zoster, for

17 example, was shown to influence the intention of general practitioners (GPs) to recommend

18 vaccination, in a study of 732 GPs from the Netherlands [14]. Similarly, the existence of

19 recommendations from professional societies was judged to be important in determining

20 vaccination in an online survey of 138 physicians in the USA [11]. Conversely, it was shown in

21 another survey of 603 primary care physicians that vaccination practices are not in compliance with

22 existing recommendations [12], and in another survey by the same group, primary care physicians

23 were found to inexplicably prioritize some vaccines over others, resulting in lost opportunities for

24 vaccination [15]. 
Furthermore, across different groups of physicians, there are wide variations in understanding, information needs and access to information. In a self-reported questionnaire survey of 346 hospital-based specialists and 335 community GPs, Ridda et al reported that hospital-based specialists tended to consider it the GPs' responsibility to provide vaccination [9]. In a web-based cohort study of 2,506 medical residents from 18 universities in Italy, the quality of training about influenza vaccination was considered fair or poor in both pre-graduate $(40.7 \%)$ and post-graduate medical training (59.6\%) [16]. In this same study, the adherence of tutors and peers to influenza vaccination recommendations, as well as correct knowledge and consultation of scientific sources about the vaccine was associated with increased uptake of influenza vaccination [16]. In turn, patients' propensity to accept $\mathrm{HZ}$ vaccination in Italy has been shown to be positively influenced by the physician's recommendation [17].

Therefore, giving healthcare providers accurate information that is likely to influence their vaccination practices and increase their propensity to recommend immunization is crucial to achieving adequate uptake on the part of the patients. This is particularly important in view of the about vaccines and vaccination in Italy.

\section{Methods}

The survey was carried out from 1 October to 30 November 2018 among qualified specialists in geriatric medicine, internal medicine and general practitioners, as well as residents training to qualify in these disciplines. Physicians and residents were recruited among the members of the 
Italian Society of Gerontology and Geriatrics (SIGG). An email with an electronic copy of the questionnaire was sent to the Directors of Internship Programs in Geriatrics $(\mathrm{N}=51)$ registered on the membership list of the SIGG, with an invitation to participate in the survey and to distribute the survey to all their colleagues and residents for participation. There were a total of 450 registered residents in training at the time of survey.

Study questionnaire

The questionnaire used was specifically designed for the purposes of this study by a consensus group comprising 5 geriatricians with specific expertise in the field, questionnaire design and vaccination. It was reviewed by two specialists in Infectious Diseases. The English translation of the questionnaire is provided in the Supplementary Appendix. The questions aimed to assess the following areas: (1) demographics and type of practice; (2) information needs and sources; (3) knowledge about the influenza, pneumococcal, and $\mathrm{HZ}$ vaccines, recommended target groups and contraindications, and practices in this area, as tested through a series of questions presenting cases where the respondent is invited to choose an answer from among multiple choice options. For each question, there was only one correct answer among multiple choices.

\section{Statistical analysis}

70 Quantitative variables are presented as mean and standard deviation (SD) or median and

71 interquartile range as appropriate according to the distribution, and categorical variables are expressed as number (percentage). The proportion who answered correctly was calculated for each question, and separately for qualified specialists and residents. The proportion of correct responders was compared between residents and qualified senior physicians for each question using the chi square test. Characteristics were compared between best performers (those with a percentage of correct responses in the highest quartile) and worst performers (those with a percentage of correct 
77 response in the lowest quartile). No imputation was applied for missing data. All analyses were

78 performed using R software, v 3.5.3 for Linux.

79

80 
A total of 472 physicians completed the survey; 245 (52\%) were females; 253 (53.6\%) were qualified specialists, 206 (43.6\%) were residents and the remaining $13(2.8 \%)$ failed to indicate whether they were qualified or residents and were excluded from further analysis. The response rate among residents was 206/450 (46\%). The characteristics of the respondents by qualification status are compared in Table $1(\mathrm{~N}=459)$. The mean age was 40.3 (SD: 12.8 , median 35, minimum 25, maximum 71 years). More than half qualified as doctors within the last 10 years, and the majority of respondents worked in acute care wards (almost 60\%). There were significant differences between specialists and residents in all characteristics, with more women, lower age and fewer years of practice on average among residents.

Regarding respondents' knowledge of vaccination, almost half responded that they used PubMed $(\mathrm{N}=216)$ and governmental websites/publications $(\mathrm{N}=241)$ as their main source of knowledge about vaccination, while 186 also reported using internet as a main source of information. Fewer respondents obtained their information from courses/congresses $(\mathrm{N}=127)$ or journals $(\mathrm{n}=126$ and 106 for international and national journal respectively), while pharmaceutical company representatives were the least popular source of information $(\mathrm{N}=57)$. Respondents reported than on average, $33 \%$ of their patients asked them for information about vaccination (median [IQR] 30 [10;50]), while $29 \%$ of carers asked about vaccination (median 20 [10;50]).

The reported barriers to advising vaccination were primarily the patient's fear of side effects (cited by 300 respondents $(66.2 \%)$ and patient skepticism about efficacy (90 (19.9\%)), while only 63 $(13.9 \%)$ reported a lack of time or information as a barrier.

Overall, $76.5 \%$ of respondents said they propose (or intend to propose) influenza vaccination, $46.3 \%$ pneumococcal vaccination, and only $24.8 \% \mathrm{HZ}$ vaccination. When asked about prescription of herpes zoster vaccination for a patient aged over 60 years, $180(41.5 \%)$ responded that they would only prescribe it if the patient had multiple comorbidities and disability, 157 (36.2\%) would 
106 prescribe it if the patient has multiple comorbidities, while $58(13.4 \%)$ would prescribe it after a

107 first episode of zoster, and only 39 (9\%) would systematically prescribe it.

108 Regarding knowledge about vaccination, the proportion of correct answers among both qualified

109 specialists and residents is shown in Table 2. There were significant differences in the proportion of 110 correct answers on 8 out of $18(44.5 \%)$ questions with residents having higher rates of correct

111 answers than qualified specialists in all cases, except for one question, where specialists scored 112 significantly better (albeit still with only $20.9 \%$ correct answers compared to $8.5 \%$ in 113 residents $(\mathrm{p}=0.0005)$.

114 Overall, the rates of correct answers were low, with more than 50\% of correct responses achieved 115 on only 5 questions out of 18 (27.8\%); for two questions, less than one out of five physicians 116 responded correctly. The rates of correct responses for each question according to the type of 117 vaccination are shown in Figure 1.

118 When comparing those with the highest scores (top quartile of correct answers; best performers) 119 and those with the lowest scores (bottom quartile of correct answers; worst performers), there were 120 significantly more men among the poor performers, who also had more years of experience on 121 average, and were slightly older than the best performers. There were significantly more residents 122 among the best performers, and more qualified specialists among the poor performers (Table 3). 
127 Our survey study among Italian specialists and residents shows that the overall level of knowledge about vaccines is quite low in both groups, although residents performed significantly better than qualified specialists on 7 out of 18 questions. Significantly more residents achieved a score in the top quartile of correct answers compared to qualified specialists. In terms of patients' awareness, only one third of patients (and even fewer carers) were reported by the physicians to enquire about vaccinations.

These findings highlight the observation that routine practices fall considerably short of recommended practices. It has long-since been established that non-negligible proportions of providers fail to recommend guidelines-advocated vaccines to their patients [18]. This may be explained in our study by the fact that the most common care setting was acute care, where management of acute events takes priority, and discussing (or administering) vaccines is of lesser importance. Significant gaps in knowledge among physicians regarding specific vaccines have previously been reported, notably low levels of knowledge about trivalent inactivated and high-dose influenza vaccines in a study of 281 providers in an urban academic medical center [19]. Improved knowledge has shown to boost the physician's own confidence, consequently affecting their willingness to recommend the vaccine to their patients [20]. Indeed, healthcare providers (HCPs) are often the most trusted advisor and hold sway with patients in their decisions to receive vaccination. A provider recommendation is a strong predictor of patient uptake [17, 21, 22]. Therefore, if the HCP is well-informed and confident, uptake may increase among patients [20], whereas there will be many lost opportunities for vaccination if the HCP is misinformed or nonknowledgeable. A further downside with greater potential for harm is that poor knowledge could even result in incorrect administration of vaccines to ineligible patients. In this regard, in an 
$15123 \%$ would incorrectly recommend a live vaccine to immunocompromised patients with

152 inflammatory bowel disease [23]. This underscores the importance of ensuring that HCPs are

153 properly informed with adequate knowledge of target groups and contraindications, and

154 recommendations for the vaccine. Indeed, for reasons that remain unclear, some providers have

155 been found to prioritize some vaccines over others, and to give lower priority to vaccines as

156 compared to other preventive interventions [15].

157

158 In our study, respondents reported that only one third of patients asked about the need for vaccination. This indicates that there is great potential for proactive intervention on the physicians' part to reach out systematically to those who do not spontaneously enquire about immunization. Among patients, common reasons for not receiving vaccines are that the patient did not know it was necessary, or believed it was not necessary because they were currently healthy [24]. In this context, physicians clearly have a major role to play in alerting patients to the need for vaccination, since, as mentioned previously, provider recommendations have concrete repercussions in terms of uptake.

In terms of the different vaccines for which knowledge was examined, residents performed better than specialists on all questions pertaining to pneumococcal vaccine and influenza vaccine, but specialists performed better on $2 / 5$ questions involving herpes zoster, and the single question on measles. However, although trainees showed higher levels of knowledge, the overall correct response rates were still low, especially for $\mathrm{HZV}$, where all the questions had correct response rates

171 below $50 \%$ (i.e. less than one physician in two could answer them correctly - no better than

172 guessing). This is in line with reports that herpes zoster vaccine lags behind influenza and 173 pneumococcal vaccines in terms of recommendations by HCPs, even though all three vaccines are 174 provided free of charge for all persons aged over 65 years in Italy. Despite the fact that providers generally agree that herpes zoster vaccination is important, they nonetheless report that more of their patients get influenza and pneumococcal vaccines [11]. In a cross-sectional internet-based 
survey administered to 266 general internal medicine physicians, 95\% and 92\% respectively reported that influenza and pneumococcal vaccines were important, compared to only $66 \%$ for herpes zoster [25]. Among patients, Lu et al reported from an internet panel survey of a nationally representative sample of U.S. adults, that herpes zoster vaccine was the least well known among American adults, while influenza was the most well known vaccine [26]. Similarly, in a survey of 4,023 Canadian adults, $77.4 \%$ reported that they were offered influenza vaccine, but only 17.8 and $7.9 \%$ respectively were offered pneumococcal and herpes zoster vaccines [13]. Clearly, there is a mismatch between the providers' assertion that vaccination is important, and how these attitudes translate into their practices in recommending it. This could be at least partially explained by the fact that the herpes zoster vaccine is the most recent of the three, and could therefore be less well known to both patients and HCPs. In this regard, there is a dearth of studies investigating provider prescribing behavior as an outcome [27], making it difficult to assess exactly how knowledge affects prescription practices.

Our findings that residents performed better than qualified specialists on most questions pertaining to vaccine knowledge are in line with previous findings. Medical students were shown to have higher levels of knowledge of influenza vaccines than nursing students, and generally, healthcare students tended to be pro-vaccination in a survey of medical, pharmacy and nursing students in Canada [28]. Conversely, in a study of 256 qualified nurses and 292 physicians in British Columbia, nurses appeared to have higher overall immunization knowledge than physicians and were more likely to receive training outside of the academic setting [29]. Educational initiatives can surely improve provider knowledge, whether among trainees or qualified specialists. Educational initiatives have previously been shown to translate into improved pneumococcal vaccination rates in a pharmacy-driven education campaign in the state of Rhode Island [30]. Similarly, among Italian students of medicine at Palermo university, educational interventions significantly improved knowledge through extracurricular seminars [31]. By their own admission, trainees themselves may 
perceive a need for further training. In a study of 385 pediatric residents in the USA, $96 \%$ reported that they would benefit from receiving more information [32]. Clearly, increased emphasis on vaccination in undergraduate medical education and during clinical residency is essential and should be integrated into the usual curriculum to prepare future generations of HCPs to promote vaccination once they become professionally active. This is particularly important in ensuring they have the skills to address such challenging situations as vaccine hesitancy, for example [33].

\section{Study limitations}

211 This study has some limitations that need to be taken into account. A first limitation concerns the sample, which was a convenience sample selected due to the ease of access through the SIGG. In

213 addition, the survey was sent only to the Directors of Internship programs, and we relied on their 214 good will to forward the survey to specialists and residents in their entourage. We therefore cannot 215 rule out the possibility that potentially eligible participants were not reached. However, after sending the survey to 51 program directors, a total of 472 completed questionnaires were returned, which attests to the active collaboration of these directors in forwarding the survey to relevant respondents. A second limitation concerns the experimental nature of the questionnaire. Indeed, the questionnaire was developed ad hoc for the purposes of this survey, and its psychometric properties were not evaluated. There may be determinant domains that could influence practice but that were not explored by this questionnaire. However, irrespective of the type of practice or profile of patients treated, the questions judging the level of knowledge remain relevant, and thus, examining knowledge is pertinent to uncover information needs amenable to educational interventions in this population. Finally, an alternative explanation of our results is that different practice settings may be driving the difference between qualified specialists and trainees, rather than the level of training. Further studies are warranted to investigate variations in levels of knowledge between practice settings. 


\section{Conclusion}

230 In conclusion, our study shows that residents in geriatric and internal medicine have a higher level

231 of vaccine knowledge than qualified specialists in these disciplines, even though overall levels of

232 knowledge were low in both groups. Both specialists and residents appeared to achieve higher rates

233 of correct responses on questions pertaining to influenza and pneumococcal vaccines than on

234 questions relating to $\mathrm{HZV}$ vaccine. Increased focus on vaccines and immunization in the education

235 of future generations of healthcare providers, and targeted interventions to improve knowledge

236 among qualified specialists in practice are avenues to explore as one path to increased vaccine

237 uptake among the general population.

238

239 


\section{$240 \quad$ Figure Legend}

241 Figure 1: Range (minimum to maximum) of correct response rates achieved on each question by 242 qualified specialists and residents, according to the vaccine mentioned in the question.

243 (Note: the single question about measles is not included) 


\section{Acknowledgements}

247 This research was partially funded by an unrestricted educational grant from Merck. 
References

[1] GBD 2017 Influenza Collaborators. Mortality, morbidity, and hospitalisations due to influenza lower respiratory tract infections, 2017: an analysis for the Global Burden of Disease Study 2017. 254 Lancet Respir Med. 2019;7:69-89.

[2] Torres A, Cilloniz C, Blasi F, Chalmers JD, Gaillat J, Dartois N, et al. Burden of pneumococcal community-acquired pneumonia in adults across Europe: A literature review. Respir Med. 2018; 137:6-13.

[3] Bricout H, Haugh M, Olatunde O, Prieto RG. Herpes zoster-associated mortality in Europe: a systematic review. BMC Public Health. 2015;15:466.

[4] Pinchinat S, Cebrian-Cuenca AM, Bricout H, Johnson RW. Similar herpes zoster incidence across Europe: results from a systematic literature review. BMC Infect Dis. 2013;13:170. [5] Amirthalingam G, Andrews N, Keel P, Mullett D, Correa A, de Lusignan S, et al. Evaluation of the effect of the herpes zoster vaccination programme 3 years after its introduction in England: a 264 population-based study. Lancet Public Health. 2018;3:e82-e90.

265 [6] Blasi F, Akova M, Bonanni P, Dartois N, Sauty E, Webber C, et al. Community-acquired 266 pneumonia in adults: Highlighting missed opportunities for vaccination. Eur J Intern Med. 2017;37:13-8.

268 [7] European Centre for Disease Prevention and Control. Seasonal influenza vaccination in Europe.

269 Vaccination recommendations and coverage rates in the EU Member States for eight influenza 270 seasons: 2007-2008 to 2014-2015. Stockholm: European Centre for Disease Prevention and 271 Control; 2017. Available at: https://ecdc.europa.eu/en/publications-data/seasonal-influenza272 vaccination-europe-vaccination-recommendations-and-coverage-2007-2015 [Access date: 4 April 273 2019] doi: 10.2900/153616. 
[8] Fogelberg S, Lamb F, Gronlund O, Eriksson I, Sundstrom K, Clements M, et al. Differential

uptake of herpes zoster vaccination associated with socioeconomic status: A population-based study in Stockholm County, Sweden. Pharmacoepidemiol Drug Saf. 2018;27:1159-65.

[9] Ridda I, Lindley IR, Gao Z, McIntyre P, Macintyre CR. Differences in attitudes, beliefs and knowledge of hospital health care workers and community doctors to vaccination of older people. Vaccine. 2008;26:5633-40.

[10] Klett-Tammen CJ, Krause G, von Lengerke T, Castell S. Advising vaccinations for the elderly: a cross-sectional survey on differences between general practitioners and physician assistants in Germany. BMC Fam Pract. 2016;17:98.

[11] Tsui E, Gillespie C, Perskin M, Zabar S, Wu M, Cohen EJ. Evaluating Physician Attitudes and Practices Regarding Herpes Zoster Vaccination. Cornea. 2018;37:947-51.

[12] Hurley LP, Allison MA, Dooling KL, O'Leary ST, Crane LA, Brtnikova M, et al. Primary care physicians' experience with zoster vaccine live (ZVL) and awareness and attitudes regarding the new recombinant zoster vaccine (RZV). Vaccine. 2018;36:7408-14.

[13] MacDougall DM, Halperin BA, MacKinnon-Cameron D, Li L, McNeil SA, Langley JM, et al.

The challenge of vaccinating adults: attitudes and beliefs of the Canadian public and healthcare 290 providers. BMJ Open. 2015;5:e009062.

[14] Lehmann BA, Eilers R, Mollema L, Ferreira J, de Melker HE. The intention of Dutch general practitioners to offer vaccination against pneumococcal disease, herpes zoster and pertussis to people aged 60 years and older. BMC Geriatr. 2017;17:122.

[15] Hurley LP, Bridges CB, Harpaz R, Allison MA, ST OL, Crane LA, et al. Physician Attitudes Toward Adult Vaccines and Other Preventive Practices, United States, 2012. Public Health Rep. 2016;131:320-30.

[16] Costantino C, Amodio E, Calamusa G, Vitale F, Mazzucco W. Could university training and a proactive attitude of coworkers be associated with influenza vaccination compliance? A multicentre survey among Italian medical residents. BMC Med Educ. 2016;16:38. 
[17] Valente N, Lupi S, Stefanati A, Cova M, Sulcaj N, Piccinni L, et al. Evaluation of the

301 acceptability of a vaccine against herpes zoster in the over 50 years old: an Italian observational study. BMJ Open. 2016;6:e011539.

[18] Nichol KL, Zimmerman R. Generalist and subspecialist physicians' knowledge, attitudes, and practices regarding influenza and pneumococcal vaccinations for elderly and other high-risk patients: a nationwide survey. Arch Intern Med. 2001;161:2702-8.

306 [19] Tewell C, Wright PW, Talbot HK. Provider knowledge of trivalent inactivated and high-dose 307 influenza vaccines. Vaccine. 2014;32:6025-8.

308 [20] Paterson P, Meurice F, Stanberry LR, Glismann S, Rosenthal SL, Larson HJ. Vaccine hesitancy 309 and healthcare providers. Vaccine. 2016;34:6700-6.

310 [21] Lu PJ, Srivastav A, Amaya A, Dever JA, Roycroft J, Kurtz MS, et al. Association of provider 311 recommendation and offer and influenza vaccination among adults aged >/=18years - United States. 312 Vaccine. 2018;36:890-8.

313 [22] Kahn KE, Black CL, Ding H, Williams WW, Lu PJ, Fiebelkorn AP, et al. Influenza and Tdap 314 Vaccination Coverage Among Pregnant Women - United States, April 2018. MMWR Morb Mortal 315 Wkly Rep. 2018;67:1055-9.

316 [23] Gurvits GE, Lan G, Tan A, Weissman A. Vaccination practices in patients with inflammatory 317 bowel disease among general internal medicine physicians in the USA. Postgrad Med J. $318 \quad 2017 ; 93: 333-7$.

319 [24] Korkmaz P, Pasali Kilit T, Onbasi K, Mistanoglu Ozatag D, Toka O. Influenza vaccination 320 prevalence among the elderly and individuals with chronic disease, and factors affecting 321 vaccination uptake. Cent Eur J Public Health. 2019;27:44-9.

322 [25] Elkin Z, Cohen EJ, Goldberg JD, Gillespie C, Li X, Jung J, et al. Studying physician 323 knowledge, attitudes, and practices regarding the herpes zoster vaccine to address perceived barriers 324 to vaccination. Cornea. 2013;32:976-81. 
[26] Lu PJ, O'Halloran A, Kennedy ED, Williams WW, Kim D, Fiebelkorn AP, et al. Awareness among adults of vaccine-preventable diseases and recommended vaccinations, United States, 2015. Vaccine. 2017;35:3104-15.

[27] Gosselin Boucher V, Colmegna I, Gemme C, Labbe S, Pelaez S, Lavoie KL. Interventions to improve vaccine acceptance among rheumatoid arthritis patients: a systematic review. Clin

$330 \quad$ Rheumatol. 2019.

331 [28] Ghandora H, Halperin DM, Isenor JE, Taylor BA, Fullsack P, Di Castri AM, et al. Knowledge, 332 attitudes, behaviours, and beliefs of healthcare provider students regarding mandatory influenza 333 vaccination. Hum Vaccin Immunother. 2019;15:700-9.

334 [29] Buxton JA, McIntyre CC, Tu AW, Eadie BD, Remple VP, Halperin B, et al. Who knows more 335 about immunization?: Survey of public health nurses and physicians. Can Fam Physician. $336 \quad 2013 ; 59: \mathrm{e} 514-21$.

337 [30] Caffrey AR, DeAngelis JM, Ward KE, Orr KK, Morrill HJ, Gosciminski M, et al. A 338 pharmacist-driven academic detailing program to increase adult pneumococcal vaccination. J Am 339 Pharm Assoc (2003). 2018;58:303-10.

340 [31] Marotta C, Raia DD, Ventura G, Casuccio N, Dieli F, D'Angelo C, et al. Improvement in 341 vaccination knowledge among health students following an integrated extra curricular intervention, 342 an explorative study in the University of Palermo. J Prev Med Hyg. 2017;58:E93-E8.

343 [32] Cordrey K, McLaughlin L, Das P, Milanaik R. Pediatric Resident Education and Preparedness 344 Regarding Vaccine-Preventable Diseases. Clin Pediatr (Phila). 2018;57:327-34.

345 [33] Caruso Brown AE, Suryadevara M, Welch TR, Botash AS. "Being Persistent without Being 346 Pushy": Student Reflections on Vaccine Hesitancy. Narrat Inq Bioeth. 2017;7:59-70. 
350 Table 1: Characteristics of the respondents to the survey about vaccination knowledge and practices

$351(\mathrm{~N}=472)$ comparing qualified specialists and residents in training.

\begin{tabular}{|c|c|c|c|c|}
\hline & All & $\begin{array}{l}\text { Specialists } \\
\qquad(\mathrm{N}=\mathbf{2 5 3})\end{array}$ & $\begin{array}{l}\text { Residents } \\
\qquad(\mathrm{n}=206)\end{array}$ & $\mathbf{P}$ \\
\hline Women [N (\%)] & $239(52.2)$ & $109(43.3)$ & $130(63.1)$ & $<0.001$ \\
\hline Age [mean (SD)] & $40.3(12.8)$ & $48.5(11.6)$ & $30.1(3.2)$ & $<0.001$ \\
\hline Years of practice [mean, $(\mathrm{SD})]$ & $13.4(12.8)$ & $21.9(11.7)$ & $3.2(1.8)$ & $<0.001$ \\
\hline Type of practice & & & & $<0.001$ \\
\hline Acute care & $264(60.7)$ & $124(49.8)$ & $140(75.3)$ & \\
\hline Outpatients & $63(14.5)$ & 47 (18.9) & $16(8.6)$ & \\
\hline Nursing home & $23(5.3)$ & $18(7.2)$ & $5(2.7)$ & \\
\hline Post-acute care & $20(4.6)$ & $11(4.4)$ & $9(4.8)$ & \\
\hline Other & $65(14.9)$ & $49(19.7)$ & $16(8.6)$ & \\
\hline Area & & & & $<0.001$ \\
\hline North & $80(18.0)$ & $28(11.5)$ & $52(25.9)$ & \\
\hline Center & $81(18.2)$ & $44(18.0)$ & $37(18.4)$ & \\
\hline South & $284(63.8)$ & $172(70.5)$ & $112(55.7)$ & \\
\hline
\end{tabular}


Table 2: Comparison of the rate of correct answers among qualified specialists and among residents in training

\begin{tabular}{|c|c|c|c|}
\hline Question & $\begin{array}{l}\text { Correct } \\
\text { answers among } \\
\text { qualified } \\
\text { specialists, } N \\
(\%)\end{array}$ & $\begin{array}{l}\text { Correct } \\
\text { answers } \\
\text { among } \\
\text { residents, N } \\
(\%)\end{array}$ & p-value \\
\hline $\begin{array}{l}1 \text { - } 71 \text { year old man, good general condition, with hypertension, atrial fibrillation, } \\
\text { hypertrophic prostate and hypothyroidism, received the pneumococcal vaccine at age } 66 \text {. } \\
\text { Correct answer: strongly indicated }\end{array}$ & 30.0 & 36.6 & 0.167 \\
\hline $\begin{array}{l}2 \text { - Irrespective of the strength and accuracy of the indication, to consolidate the effect } \\
\text { of this vaccine, it would now be necessary to... Correct answer: take no further action }\end{array}$ & 23.3 & 38.8 & 0.0004 \\
\hline $\begin{array}{l}3 \text { - If we wanted to increase vaccine coverage, should we use the 23-valent } \\
\text { pneumococcal vaccine? Correct answer: Yes, because the sequence "PCV13 then } \\
\text { PPSV23" is optimal }\end{array}$ & 59.3 & 71.0 & 0.0170 \\
\hline $\begin{array}{l}4 \text { - In the presence of chronic corticosteroid therapy, pneumococcal vaccination is.... Correct } \\
\text { answer: Efficacious, regardless of therapy }\end{array}$ & 65.2 & 70.6 & 0.263 \\
\hline
\end{tabular}




\begin{tabular}{|c|c|c|c|}
\hline $\begin{array}{l}5 \text { - In a patient with systemic lupus erythematosus, the addition of belimumab on top of an } \\
\text { alkylating agent... Correct answer: would not reduce the response to influenza vaccination }\end{array}$ & 32.5 & 35.5 & 0.584 \\
\hline $\begin{array}{l}\text { - Concomitant presence of COPD, renal insufficiency and diabetes mellitus. Correct } \\
\text { answer: clear indications for both influenza and pneumococcal vaccines. }\end{array}$ & 5.5 & 15.0 & 0.0011 \\
\hline $\begin{array}{l}7 \text { - The 23-valent polysaccharide pneumococcal vaccine.... Correct answer: includes } \\
\text { PCV13 }\end{array}$ & 73.0 & 82.5 & 0.023 \\
\hline $\begin{array}{l}8 \text { - In a } 77 \text { year old man in good general condition, herpes zoster vaccination is } \\
\text { indicated... Correct answer: always }\end{array}$ & 30.8 & 49.3 & 0.0001 \\
\hline $\begin{array}{l}9 \text { - Persisting symptoms one month after onset of herpes zoster.... Correct answer: is not a } \\
\text { contraindication to vaccination }\end{array}$ & 41.6 & 35.3 & 0.207 \\
\hline $\begin{array}{l}10 \text { - What form of bacterial pneumonia is predominantly prevented by influenza } \\
\text { vaccination? Correct answer: Staphylococcus }\end{array}$ & 7.2 & 12.8 & 0.0651 \\
\hline $\begin{array}{l}11 \text { - Simultaneous administration of influenza and pneumococcal vaccines is... Correct } \\
\text { answer: possible }\end{array}$ & 62.7 & 67.3 & 0.350 \\
\hline $\begin{array}{l}12 \text { - With respect to measles, a subject born in } 1945 \text { should be considered... Correct } \\
\text { answer: immune }\end{array}$ & 20.9 & 8.5 & 0.0005 \\
\hline 13 - The simultaneous administration of herpes zoster and 23-valent pneumococcal & 19.1 & 35.3 & 0.0003 \\
\hline
\end{tabular}




\begin{tabular}{|c|c|c|c|}
\hline $\begin{array}{l}\text { vaccines... Correct answer: Could reduce the immunogenic potential of the herpes } \\
\text { zoster vaccine }\end{array}$ & & & \\
\hline $\begin{array}{l}14 \text { - In an immunosuppressed individual.... Correct answer: Both herpes zoster and live } \\
\text { attenuated influenza vaccines are contra-indicated }\end{array}$ & 45.3 & 49.0 & 0.491 \\
\hline $\begin{array}{l}15 \text { - In the presence of an allergy to egg protein.... Correct answer: influenza vaccination is } \\
\text { contra-indicated }\end{array}$ & 43.6 & 46.5 & 0.610 \\
\hline $\begin{array}{l}16 \text { - According to the national vaccine schedule, the minimal influenza vaccine coverage } \\
\text { should be.... Correct answer: }>75 \% \text { all of the time }\end{array}$ & 39.9 & 43.6 & 0.484 \\
\hline $\begin{array}{l}17 \text { - Pneumococcal vaccination for the elderly can be proposed.... Correct answer: At } \\
\text { any time of year, and together with influenza vaccine }\end{array}$ & 62.4 & 76.7 & 0.0014 \\
\hline $\begin{array}{l}18 \text { - Regarding herpes zoster vaccination, the statement that "coverage does not decline } \\
\text { with increasing age of the vaccinated subjects" is untrue, and therefore, the correct answer }\end{array}$ & 31.3 & 28.9 & 0.657 \\
\hline
\end{tabular}

Questions with significant differences in the proportion of correct answers between specialists and residents are highlighted in bold. 
Table 3: Comparison of characteristics between worst performers (lowest quartile of correct answers) and best performers (highest quartile of correct answers)

\begin{tabular}{|c|c|c|c|}
\hline Variable & $\begin{array}{l}\text { Worst performers } \\
\qquad(\mathrm{N}=115)\end{array}$ & $\begin{array}{l}\text { Best performers } \\
\qquad(\mathrm{N}=114)\end{array}$ & p-value \\
\hline Women & $46(40 \%)$ & $69(60.5 \%)$ & $<0.0001$ \\
\hline Age (years) & $41.9 \pm 13.1$ & $37.6 \pm 11.2$ & $<0.001$ \\
\hline Number of years of experience & $14.1 \pm 13$ & $10.8 \pm 11.4$ & 0.015 \\
\hline Type of practice setting & & & $<0.0001$ \\
\hline Acute care & $60(54.1 \%)$ & $75(70.8 \%)$ & \\
\hline Ambulatory & $26(23.4 \%)$ & $9(8.5 \%)$ & \\
\hline Post-acute care & $3(2.7 \%)$ & $8(7.5 \%)$ & \\
\hline Other & $12(10.8 \%)$ & $10(9.4 \%)$ & \\
\hline Qualification & & & $<0.0001$ \\
\hline Qualified specialists & $73(63.5 \%)$ & $46(40.4 \%)$ & \\
\hline Residents in training & $42(36.5 \%)$ & $68(59.6 \%)$ & \\
\hline Geographical location & & & 0.001 \\
\hline Southern Italy & $75(68.6 \%)$ & $53(48.2 \%)$ & \\
\hline Central Italy & $20(18.3 \%)$ & $20(18.2 \%)$ & \\
\hline Northern Italy & $14(12.8 \%)$ & $37(33.6 \%)$ & \\
\hline
\end{tabular}




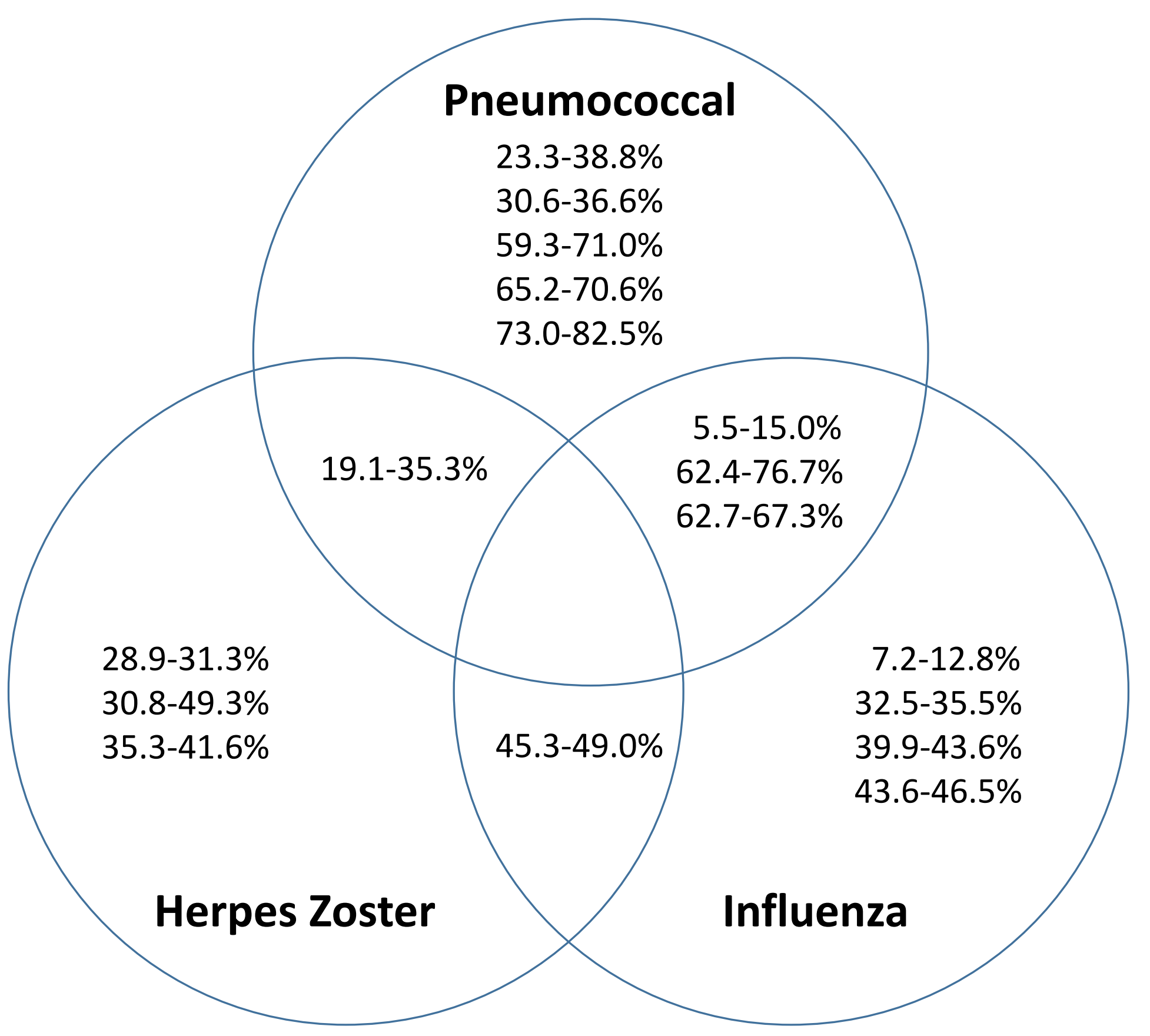

